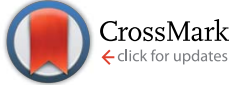

Cite this: RSC Adv., 2017, 7, 13868

Received 23rd December 2016 Accepted 17th February 2017

DOI: 10.1039/c6ra28599f

rsc.li/rsc-advances

\section{Highly efficient one-pot three-component Betti reaction in water using reverse zinc oxide micelles as a recoverable and reusable catalyst $\uparrow$}

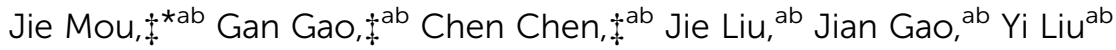 \\ and Dongsheng Pei ${ }^{\star c}$
}

An efficient synthesis of Betti bases via a one-pot three-component reaction of 2-naphthol, substituted aldehydes and anilines in aqueous media is reported. Through screening different catalysts, reverse zinc oxide nanomicelles show good activity and high selectivity. The catalyst retained activity after six cycles of reuse and were recoverable. The method has the advantages of atom economy, short reaction time, good yield, and easy work-up as well as being environmental friendly.

\section{Introduction}

The Betti reaction, which is a typical Mannich reaction, is one of the most important fundamental carbon-carbon bond-forming reactions in organic chemistry. ${ }^{1}$ The Betti reaction provides $1-(\alpha-$ aminoalkyl)-2-naphthols, the so-called Betti bases. The optical Betti base isomers are valuable ligands in asymmetric synthesis. ${ }^{2,3}$ Recently, the importance of Betti bases was well established in pharmaceutical chemistry because of their bioactivities, including anti-bacterial, antipain, antihypertensive and bradycardiac activities. $^{4-6}$ The original Betti reaction is between an aldehyde, ammonia or urea and $\beta$-naphthol in an ethanolic solution of potassium hydroxide in two steps for a long time (9-36 h). ${ }^{7,8}$ However, the synthetic approach is plagued by a number of serious disadvantages and has limited applications. Therefore, numerous modern versions of Betti reaction have been developed to overcome the drawbacks of the classical method. In general, the preferable route is the use of a one-pot three-component strategy that allows for a wide range of structural variations by using quilinols, ${ }^{9}$ naphthols ${ }^{\mathbf{1 0}}$ and alkyl amines to exchange ammonia. ${ }^{11}$ However, these methods suffered from severe side reactions and longer reaction times with lower yields.

In terms of green chemistry, the development and application of multi-component coupling reactions in water is favourable, as they provide simple and rapid access to a large number of organic molecules via a tolerable method. ${ }^{12,13}$ But the Betti

${ }^{a}$ School of Pharmacy, Xuzhou Medical University, Xuzhou 221006, China

bjiangsu Key Laboratory of New Drug and Clinical Pharmacy, Xuzhou Medical University, Xuzhou 221006, China

'Department of Pathology, Xuzhou Medical University, Xuzhou 221006, China. E-mail: dspei@xzhmu.edu.cn

$\dagger$ Electronic supplementary information (ESI) available. See DOI: $10.1039 /$ c6ra28599f

\$ Co-first authors. reaction is difficult to be initiated in water because the activity of amine is inhibited by the strong hydrogen bond with water. In recent years, rare earth and lanthanide triflates, ${ }^{14}$ sulfanilic acid-functionalized silica-coated magnetite nanoparticles, ${ }^{15}$ surfactants $^{16}$ or iminium salts ${ }^{17,18}$ as catalysts for direct-type Mannich reactions in water or under solvent-free conditions have been successfully applied. ${ }^{19,20}$ In most cases the catalysts are not used friendly and non-recoverable. To our knowledge, it is still a challenge for aromatic primary amines are employed as the substrates in water for their electron-withdrawing properties and steric hindrance. As a consequence, it is necessary to develop environmentally benign reactions and atom-economic catalytic processes for Betti reaction.

Nanocrystalline metal oxides find excellent application as active adsorbent for gas, for destruction of hazardous chemicals, and as catalyst in various organic reactions. ${ }^{21}$ Karmakar et al. reported an efficient method for the preparation of Betti base derivatives over nanocrystalline $\mathrm{MgO}$ in water. ${ }^{22}$ Unfortunately, the yield of aniline's product is lower than $20 \%$. Moreover, nanocrystalline metal oxides usually undergo agglomeration when dispersed in solutions because of large specific surface area and high surface activity. This behavior has a major impact on their reactivity. ${ }^{23}$

Under the inspiration of metallomicelles, ${ }^{\mathbf{2 4 , 2 5}}$ which were utilized for their certain similarities with the natural enzymes, we designed reverse zinc oxide nanomicelles as functional catalyst to meet the dual requirements of the disperse stability and nano-size control. Reverse micelles have been depicted as passive nano-reactors that via their shapes template the growing crystalline nuclei into narrowly dispersed or even perfectly uniform nano-sized particles. Reverse micellar systems containing metal oxide could possibly afford high local reactant concentrations near the catalysts, helping enhance the catalytic rate. ${ }^{26}$ 
In this study, direct-type three-component Betti reaction using water as the only solvent at room temperature catalyzed by reverse $\mathrm{ZnO}$ nanomicelles is reported. Reverse $\mathrm{ZnO}$ nanomicelles are prepared starting from zinc acetate as a precursor, triethanolamine as the base and hexadecyl trimethyl ammonium bromide as the surfactant in cyclohexane. This route has the advantages of atom-economy, operational-simplicity, ecofriendliness and cost-efficiency.

\section{Experimental section}

\section{Materials}

Unless otherwise noted, all reagents and solvents were purchased from commercial suppliers and used without further purification.

Preparation of reverse zinc oxide nanomicelles. Hexadecyl trimethyl ammonium bromide (CTAB, $0.3644 \mathrm{~g}, 1 \mathrm{mmol}$ ) was dissolved in cyclohexane $(10 \mathrm{~mL})$. The suspension was heated at $80{ }^{\circ} \mathrm{C}$ for $1 \mathrm{~h}$ and cooled to room temperature under stirring. After being stirred overnight, triethanolamine $(5 \mathrm{~mL})$ was added dropwise under vigorous stirring until the milk white emulsion was formed. Zinc acetate $\left(\mathrm{Zn}\left(\mathrm{CH}_{3} \mathrm{COO}\right)_{2} \cdot 2 \mathrm{H}_{2} \mathrm{O}\right)(2.19 \mathrm{~g}, 1 \mathrm{mmol})$ was dissolved in distilled water $(100 \mathrm{~mL})$ whilst stirring to obtain the $1 \mathrm{~mol} \mathrm{~L}^{-1}$ zinc solution. Zinc acetate solution $(0.2$ $\mathrm{mL}, 1 \mathrm{~mol} \mathrm{~L}^{-1}$ ) was injected into the above mentioned emulsion slowly under moderate stirring until the mixture turn cleared and stirred for another $1 \mathrm{~h}$. Then the solution was sonicated at $200 \mathrm{~W}$ for $15 \mathrm{~min}$ in an ice bath. The reacted solution kept clear, and the reverse $\mathrm{ZnO}$ micelles were formed in solution and stabilized by surfactant.

Synthesis of Betti base derivatives. The catalytic activity of the reverse $\mathrm{ZnO}$ nanomicelles was investigated by measuring their ability to synthesis Betti base derivatives. To a mixture of aromatic aldehydes ( $1 \mathrm{mmol}$ ), $\beta$-naphthol ( $1 \mathrm{mmol}, 0.144 \mathrm{~g}$ ) and aniline ( $1 \mathrm{mmol}, 0.094 \mathrm{~g}$ ) in $10 \mathrm{~mL}$ water in a round bottom flask, catalytic amount of reverse $\mathrm{ZnO}$ nanomicelles was added and stirred at room temperature for appropriate time. After completion of the reaction as monitored by thin layer chromatography (TLC), the aqueous layer was decanted to recycle the catalyst for another reaction. The crude product was filtered and purified by recrystallization from ethanol (95\%). The novel products were characterized by ${ }^{1} \mathrm{H}$ NMR, ${ }^{13} \mathrm{C}$ NMR, IR and HRMS.

Characterization methods. Dynamic light scattering (DLS) measurements were performed on a NICOMP 380ZLS zeta potential/particle size analyzer (PSS, USA) equipped with a HeNe laser at a wavelength of $660 \mathrm{~nm}$. The experimental data were analyzed by the CONTIN method, which is based on an inverseLaplace transformation of the data and provides access to a size distribution histogram for the analyzed micellar solutions. The

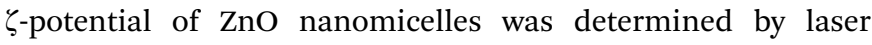
Doppler anemometry using a NICOMP 380ZLS.

Transmission electron microscopy (TEM) was performed on JEOL JEM 1230 (Joel, Japan) at an acceleration voltage of $120 \mathrm{kV}$. Then a drop of the reverse $\mathrm{ZnO}$ nanomicelle sample was applied onto a carbon-coated copper TEM grid. After solvent evaporation $\mathrm{ZnO}$ micelles remained dispersed on the carbon film of the TEM grid.
X-ray diffractometer were employed on Rigaku D/max-III type instrument (Denki, Japan) using $\mathrm{Cu} \mathrm{K} \alpha(1.54 \AA$ A ) radiation at a beam voltage $40 \mathrm{kV}$ and a $30 \mathrm{~mA}$ beam current. The patterns were obtained by continuous scans in a $2 \theta$ range of $5-90^{\circ}$ with a scan rate of $4 \mathrm{deg} \min ^{-1}$ and step $0.02 \mathrm{deg}$. XRD sample was obtained by centrifugation, washing, drying the micelles in vacuum at $70{ }^{\circ} \mathrm{C}$ in the vacuum drying oven.

Analytical thin layer chromatography (TLC) was performed using glass backed TLC extra hard layer pre-coated with silica gel (0.25 mm, 60 A pore size) and visualized by exposure to ultraviolet light (254/366 nm) using a Camag UV Lamp (Switzerland).

Proton nuclear magnetic spectra $\left({ }^{1} \mathrm{H} \mathrm{NMR}, 400 \mathrm{MHz}\right)$ and carbon nuclear magnetic resonance spectra $\left({ }^{13} \mathrm{C}\right.$ NMR, 100 $\mathrm{MHz}$ ) were recorded on an JNM-ECZ400s/L spectrometer (Joel, Japan) using $\mathrm{CDCl}_{3}$ or DMSO- $d_{6}$ as a solvent and TMS as an internal standard. IR spectra were recorded on a Jasco FT-IR 4100 Series spectrophotometer (Perkin-Elmer, USA), $v$ max $\left(\mathrm{cm}^{-1}\right)$ are partially reported. HRMS analyses were carried out using a Bruker micrOTOF-Q instrument (Bruker, USA).

\section{Results and discussion}

Preparation and characterization of reverse $\mathrm{ZnO}$ nanomicelles

Reverse $\mathrm{ZnO}$ nanomicelles were prepared according to the modified sol-gel based procedures ${ }^{27}$ using zinc acetate dehydrate as precursor and cyclohexane as solvent. Reverse micelles were formed by the self-assembly of the surfactant in apolar solvents. Metal salts are solubilized within the interior of inverse micelles where were shield by the hydrophilic head groups of the surfactant from the oleic surroundings. ${ }^{28}$ Ionic surfactants as amphiphilic molecules are often used to form reverse micelles for protein solubilization, such as anionic di-2-ethylhexyl sodium sulfosuccinate $(\mathrm{AOT})^{29}$ and cationic hexadecyl trimethyl ammonium bromide (CTAB). ${ }^{30}$ In this case, CTAB was employed in the preparation of reverse micelles. Its relatively polar head group regions solubilize and confine added zinc salts and act as reaction cages when forced hydrolysis is initiated. The reaction sequence is sustained by material exchange between reverse micelles and final particle size is dependent on stabilization by the surfactant. In our study, triethanolamine was used as base to maintain $\mathrm{pH}$ value and sonication was applied to initiate the hydrolysis of zinc ions. The reverse $\mathrm{ZnO}$ nanomicelles were formed which were sterically stabilized in solution by surfactant (Scheme 1).

The particle size and distribution of reverse $\mathrm{ZnO}$ nanomicelles was measured with NICOMP 380ZLS and the morphology was characterized using transmission electron microscopy (TEM). The distribution of sphere diameters is shown in Fig. 1. The image revealed that the average diameter was $120 \mathrm{~nm}$ and the size distribution was $80 \%$.

Fig. 2 illustrates the transmission electron microscopy (TEM) micrograph of the reverse $\mathrm{ZnO}$ nanomicelles. It can be observed that the particles are highly dispersed, subsphaeroidal and monodisperse on the TEM grid. The size range of them is approximate $200 \mathrm{~nm}$. The particles displayed a homogeneous size distribution and the particle diameters ranged from 20 to $500 \mathrm{~nm}$, as evidenced by Zata-dimeter measurement. 


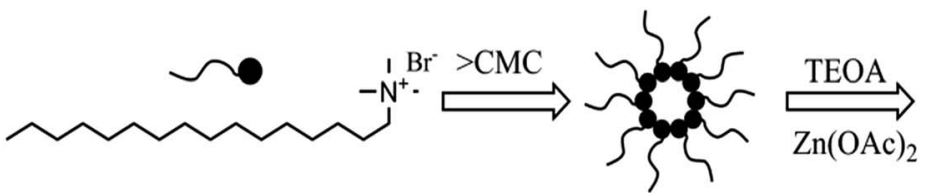

Hexadecyl Trimethyl Ammonium Bromide (CTAB)
CTAB

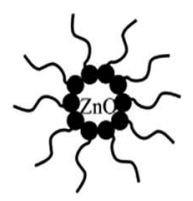

CTAB-TEOA

Nano Reverse Micelle

$$
\mathrm{Zn}^{2+}+\mathrm{H}_{2} \mathrm{O} \longrightarrow \mathrm{Zn}(\mathrm{OH})_{2} \stackrel{\text { sonication }}{\longrightarrow} \mathrm{ZnO}
$$

Scheme 1 Preparation process of reverse $\mathrm{ZnO}$ nanomicelles.

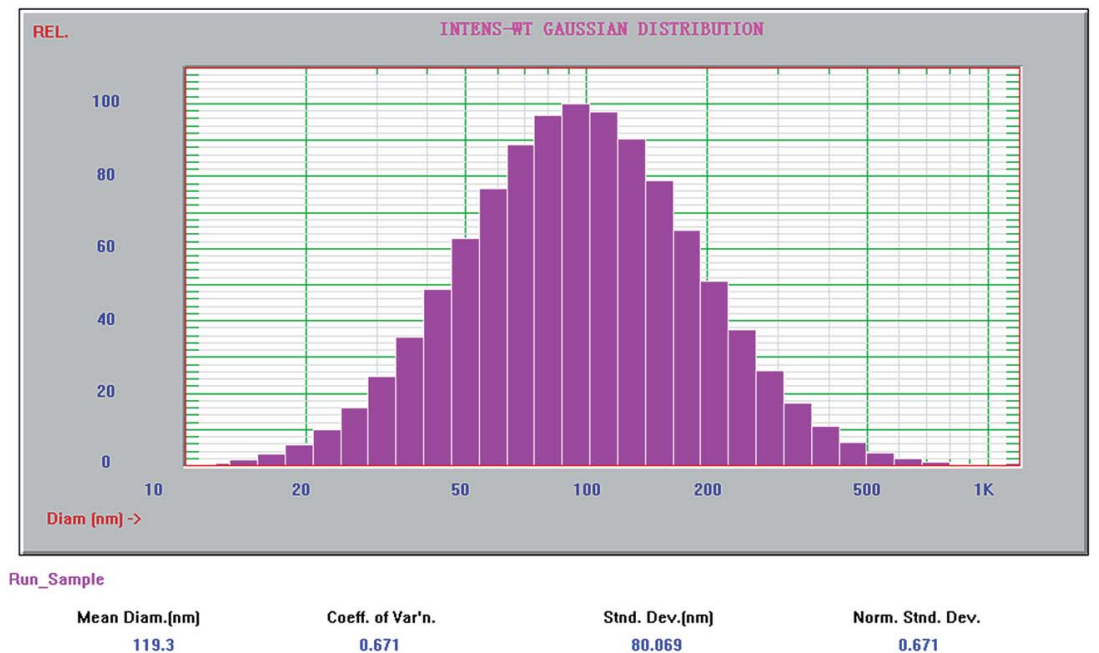

Fig. 1 Size distribution for reverse $\mathrm{ZnO}$ nanomicelles with average diameter of $120 \mathrm{~nm}$.

The formation of $\mathrm{ZnO}$ nanomicelles was investigated by $\mathrm{X}$ ray diffraction (XRD). The XRD pattern of $\mathrm{ZnO}$ nanomicelles after centrifugation, washing and drying was shown in Fig. 3.

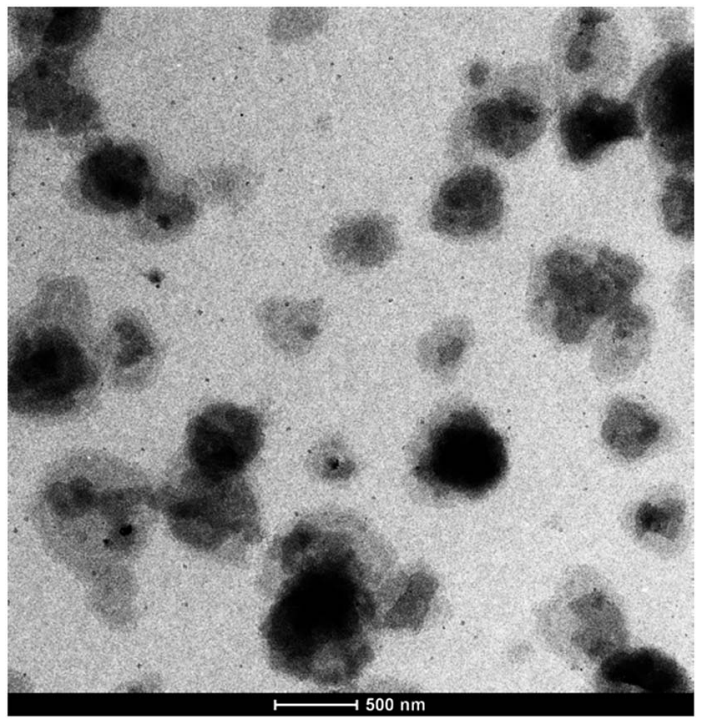

Fig. 2 TEM image of reverse $\mathrm{ZnO}$ nanomicelles.
The peaks appeared at $2 \theta$ value ranging from $31.73^{\circ}, 34.38^{\circ}$, $36.22^{\circ}, 47.50^{\circ}, 56.56^{\circ}, 62.81^{\circ}, 66.34^{\circ}, 67.91^{\circ}, 69.03^{\circ}, 72.6^{\circ}$ and $76.90^{\circ}$ values corresponded to pure ZnO. All XRD diffraction peaks of $\mathrm{ZnO}$ micelles are shown in a good agreement with hexagonal structure of zincite phase reported in JCPDS file card no. 05-0664. No peaks of impurity are observed, indicating that $\mathrm{ZnO}$ precursor has been completely transformed into nano $\mathrm{ZnO}$. The result indicated that the sonication step is a key factor in avoiding the fast precipitation and achieving a pure zinc oxide.

\section{Catalyst screening}

Initially, the preparation of $4 \mathrm{a}$ from $m$-nitrobenzaldehyde (1 equiv.), naphthol (1.2 equiv.) and aniline (1 equiv.) in water was investigated as a model system to search for the effective and reusable catalytic system (Table 1).

It was found that the reaction failed in the absence of catalyst from room temperature to $90{ }^{\circ} \mathrm{C}$ for $24 \mathrm{~h}$. Conventional basic or acidic catalyst such as sodium hydroxide, triethylamine and acetic acid only afforded Schiff base $6 \mathrm{a}$ as the terminal products (Table 1, entry 1-4). Typical Lewis acid catalysts such as $\mathrm{ZnCl}_{2}$ and $\mathrm{FeCl}_{3}$ provided bis product 5a as a major product in low yield whereas, 4 a was formed as a minor product $(<10 \%$ yield $)$ 


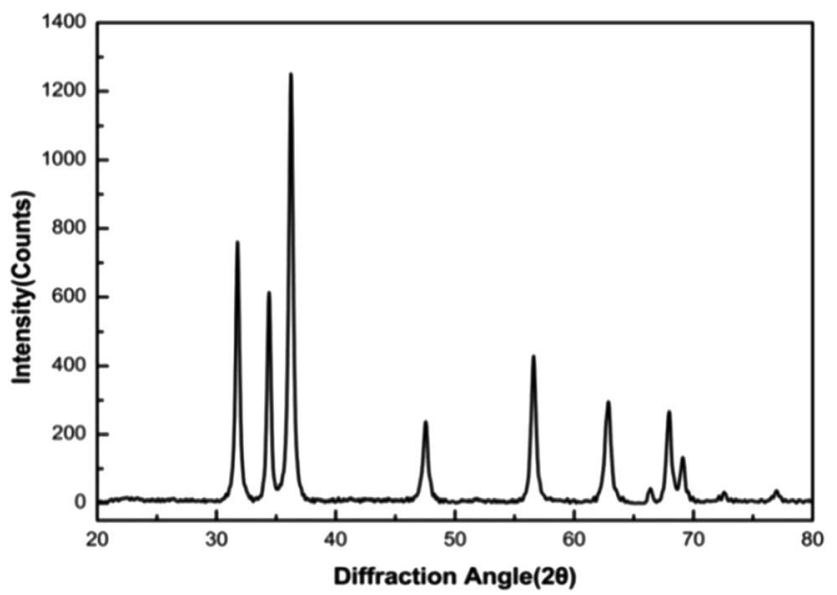

Fig. 3 XRD graph of $\mathrm{ZnO}$ nanomicelle particles.

(Table 1, entry 5 and 6). In contrast, same test reaction was carried out with different supported Lewis acid catalysts such as $\mathrm{ZnCl}_{2}-\mathrm{SiO}_{2}, \mathrm{BF}_{3}-\mathrm{SiO}_{2}$ and $\mathrm{TiO}_{2}-\mathrm{SiO}_{2}$, the yields of desired product 4 a were increased from $23 \%$ to $42 \%$ (Table 1 , entry $7-9$ ).

To study the role of nanomicelles, the reaction was performed with surfactants and $\mathrm{ZnO}$ nanoparticles (ZnO NPs). Surprisingly, the reaction was not successful either with nonionic surfactants (i.e. PEG200-PEG2000) or with ionic surfactants (i.e. CTAB). Even though the mixture of $\mathrm{ZnCl}_{2}$ and CTAB was added, there was no appreciable improvement in yield (Table 1, entry 10-13). Meanwhile, it was found that the smaller ZnO NPs $(10 \mathrm{~nm})$ can afford the desired product $4 \mathrm{a}$, which is better than ZnO NPs (100 nm) (Table 1, entry 14 and 15). The best result showed that exposure of the reaction mixture to $10 \mathrm{mmol} \%$ reverse $\mathrm{ZnO}$ nanomicelles $(100 \mathrm{~nm})$ in 10 $\mathrm{mL}$ water at $70{ }^{\circ} \mathrm{C}$ for 30 min provided 4 a with excellent yield (Table 1, entry 16). The larger size of nanomicelles $(>500 \mathrm{~nm})$ did not give more satisfied results (Table 1 , entry 18). The normal $\mathrm{ZnO}$ nanomicelles catalyst is neither activity nor selectivity to Betti reaction. Obviously, Betti reaction is sensitive to the structure of the catalyst. All the results can be explained as follows. In the catalytic process, the catalyst consists of small metal oxide particles dispersed on an inert support which was the key components of the catalyst. The activity of the catalyst will generally depend on the size of the metal oxide particles (active sites), where a catalyst with small particles will give high activity due to the large number of atoms available on the metal particles surfaces. ${ }^{31}$

\section{Solvent screening}

To investigate the feasibility of the strategy, various solvents such as dichloromethane, tetrahydrofuran, acetonitrile, ethanol and dimethyl formamide were used to improve the yield of $4 \mathrm{a}$

Table 1 Comparison of efficiency of various catalysts in one-pot synthesis of $4 a$

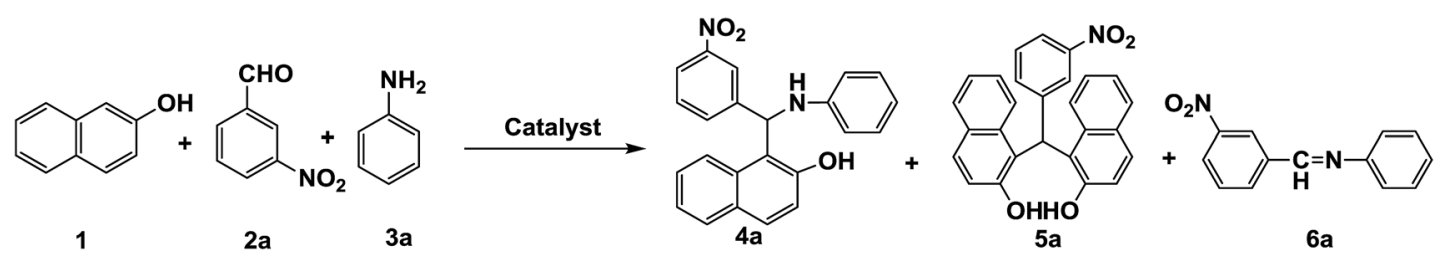

\begin{tabular}{|c|c|c|c|c|c|c|}
\hline Entry & Catalyst $^{a}$ & $T\left({ }^{\circ} \mathrm{C}\right)$ & Time (h) & 4 a yield ${ }^{b}(\%)$ & 5 a yield ${ }^{b}(\%)$ & 6 a yield ${ }^{b}(\%)$ \\
\hline 1 & $20 \% \mathrm{NaOH}$ & 90 & 8 & - & 60 & - \\
\hline 2 & $40 \% \mathrm{NaOH}$ & 90 & 8 & - & 20 & 40 \\
\hline 4 & $\mathrm{Et}_{3} \mathrm{~N}$ & 90 & 8 & - & & - \\
\hline 5 & $\mathrm{FeCl}_{3}$ & 90 & 8 & 4 & 65 & - \\
\hline 6 & $\mathrm{ZnCl}_{2}$ & 90 & 8 & 7 & 61 & - \\
\hline 9 & $\mathrm{TiO}_{2}-\mathrm{SiO}_{2}$ & 90 & 8 & 42 & 41 & - \\
\hline 10 & PEG-200 & 90 & 8 & - & - & - \\
\hline 11 & PEG-2000 & 90 & 8 & - & - & - \\
\hline 12 & CTAB & 90 & 8 & - & - & - \\
\hline 13 & $\mathrm{ZnCl}_{2}+\mathrm{CTAB}$ & 90 & 8 & Trace & 47 & Trace \\
\hline 14 & ZnO NPs $(10 \mathrm{~nm})$ & 90 & 1 & 43 & & \\
\hline 19 & Normal ZnO micelles $(100 \mathrm{~nm})$ & 70 & 8 & 27 & 19 & - \\
\hline
\end{tabular}

${ }^{a}$ Conditions: 1 (1.2 mmol), 2a (1 mmol), 3a (1 mmol), catalyst (3-6) (15 mol\%), (7-11) (0.01 g mmol ${ }^{-1}$ substrate), (12-20) (10 mol\%), solvent (water, $10 \mathrm{~mL}), 0.5-24$ h. ${ }^{b}$ Isolated yield. 
Table 2 Effects of solvent on the synthesis of $4 a^{a}$

\begin{tabular}{|c|c|c|c|c|c|}
\hline Entry & Solv. ${ }^{a}$ & Temp. $\left({ }^{\circ} \mathrm{C}\right)$ & Time (h) & 4a yield ${ }^{b}(\%)$ & 5 a yield ${ }^{b}(\%)$ \\
\hline 1 & $\mathrm{CH}_{2} \mathrm{Cl}_{2}$ & 25 & 24 & 7 & 14 \\
\hline 2 & $\mathrm{CH}_{3} \mathrm{CN}$ & 60 & 24 & 12 & 26 \\
\hline 3 & $\mathrm{THF}$ & 60 & 8 & 6 & 12 \\
\hline 4 & DMF & 80 & 8 & 34 & 28 \\
\hline 5 & EtOH & 60 & 8 & 46 & 22 \\
\hline 6 & Water & 25 & 0.15 & 87 & - \\
\hline 7 & Water & 70 & 1 & 96 & - \\
\hline
\end{tabular}

${ }^{a}$ Reactions were carried out in solvent $5.0 \mathrm{~mL}$ on a $1 \mathrm{mmol}$ scale at the special temperature using $10 \mathrm{~mol} \%$ reverse $\mathrm{ZnO}$ nanomicelles as catalyst. ${ }^{b}$ Isolated yield.

and reduce the formation of $5 \mathrm{a}$. In polar non-proton solvents such as $\mathrm{CH}_{2} \mathrm{Cl}_{2}$, THF and $\mathrm{CH}_{3} \mathrm{CN}$, both $4 \mathrm{a}$ and $5 \mathrm{a}$ were obtained in poor yield. However, in polar protic solvents like $\mathrm{C}_{2} \mathrm{H}_{5} \mathrm{OH}$ and DMF, 5a was obtained in higher yield in comparison to 4 . It was found that ethanol was superior to other examined solvents in view of yield (Table 2, entry 5). However, with further consideration on the development of environment-friendly chemistry, we tried to conduct Betti reaction in water. To our delight, when the reaction was performed in water at room temperature, only product $4 \mathrm{a}$ was formed. With further studies by increasing reaction temperature, the desired product 4 a could be obtained smoothly in $87 \%$ yield in a shortened reaction time $8 \mathrm{~min}$ (Table 2 , entry 6). The results revealed that the reaction in aqueous media condition produced the product in shortest reaction time and highest yield (96\%). Previously, reversible dehydration condensation in water is in principle a difficult transformation because the large excess of water pushes the equilibrium in favor of the hydrated compounds. ${ }^{32}$ To date, dehydration reactions in water have usually been achieved by employing the novel catalyst. ${ }^{33-38}$ In this case, it was assumed that water can favor the nucleophilic addition reaction between imine intermediate and $\beta$-naphthol for the high polarity.

\section{Surfactant screening}

To elucidate the role of nanomicelles, various non-ionic and ionic surfactants ( $5 \mathrm{~mol} \%$ ) based $\mathrm{ZnO}$ reverse micelles (5 mol\%) were tested on the reaction of $m$-nitrobenzaldehyde, naphthol and aniline in water. The results are summarized in Table 3. The reaction was run in the presence of TX 10 at room temperature for $8 \mathrm{~h}$, as a result, only very low yield of product $4 \mathrm{a}$

Table 3 Effect of surfactant-based reverse micelle on synthesis of $4 a^{a}$

\begin{tabular}{lllll}
\hline Entry & Surfactant & Catalyst & Time $(\mathrm{h})$ & 4a yield $^{b}(\%)$ \\
\hline 1 & TX10 & ZnO & 8 & - \\
2 & DBSA & ZnO & 48 & 19 \\
3 & SDS & ZnO & 48 & 23 \\
4 & CTAB & ZnO & 0.15 & 96
\end{tabular}

${ }^{a}$ Conditions: 1 (1.2 mmol), 2a (1 mmol), 3a (1 mmol), surfactant (1-4) (5 mol\%), catalyst (ZnO NPs, $5 \mathrm{~mol} \%$ ), solvent (water, $10 \mathrm{~mL}$ ), room temperature, 9 min to $48 \mathrm{~h} .{ }^{b}$ Isolated yield. was obtained. In the presence of $p$-dodecylbenzenesulfonic acid (DBSA) and sodium dodecyl sulfate (SDS), which acts both Bronsted acid and anionic surfactant, only $19 \%$ and $23 \%$ of desired product $4 \mathrm{a}$ was gained. CTAB acts as cationic surfactant. CTAB based reverse $\mathrm{ZnO}$ nanomicelles proved to be the best choice. It was known that the reverse micelles are small molecular assemblies that encapsulate nanoscopic pools of water. ${ }^{39}$ The results revealed that the interaction of the cationic surfactant head groups and their $\mathrm{Br}^{-}$counter ions in water pool is believed to play a major role in controlling the yields by promoting the dehydration condensation in water.

\section{Stability of the reaction system}

The effect of reaction temperature was carefully examined (Fig. 4). The reaction was smoothly promoted with the increasing of temperature from room temperature to $70{ }^{\circ} \mathrm{C}$. After 9 minutes, the highest yield was obtained at $70{ }^{\circ} \mathrm{C}$. However, the catalyst activity declined as the reaction temperature increased over $70{ }^{\circ} \mathrm{C}$. Loss in surface area caused by the dissociation of the micelles could be the reason for the decreased activity. The effect of reaction time was also investigated (Fig. 5). It was found that the reaction was started $2 \mathrm{~min}$ later and maintained a stable state for a long period. The yield did not increase significantly from $8 \mathrm{~min}$ to $20 \mathrm{~min}$.

\section{Recyclability of catalyst}

Additionally, recycle and reusability of the catalyst was examined (Table 4). After the completion of the reaction, the aqueous layer was decanted and the catalyst was reused for the next run under the same conditions. As shown in Table 4, the desired products yields remained essentially constant for the six successive cycles, reflecting high stability and reusability of the catalyst. This is consistent with the characterization results for this catalyst. Dynamic light scattering (DLS) measurements

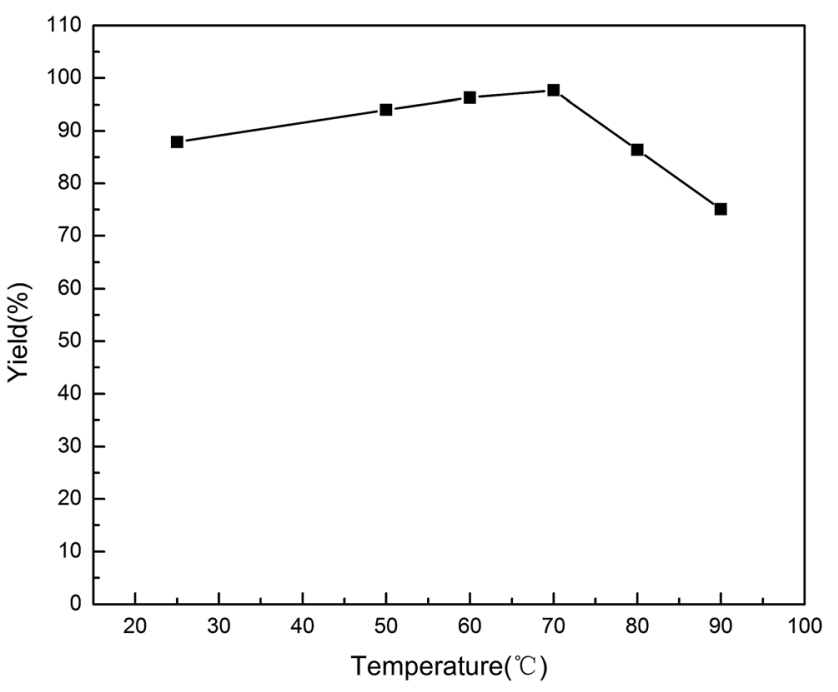

Fig. 4 Influence of reaction temperature on synthesis of 4 a. Reactions were carried out on a $0.1 \mathrm{mmol}$ scale; reverse $\mathrm{ZnO}$ nanomicelles 20 mol\%. 


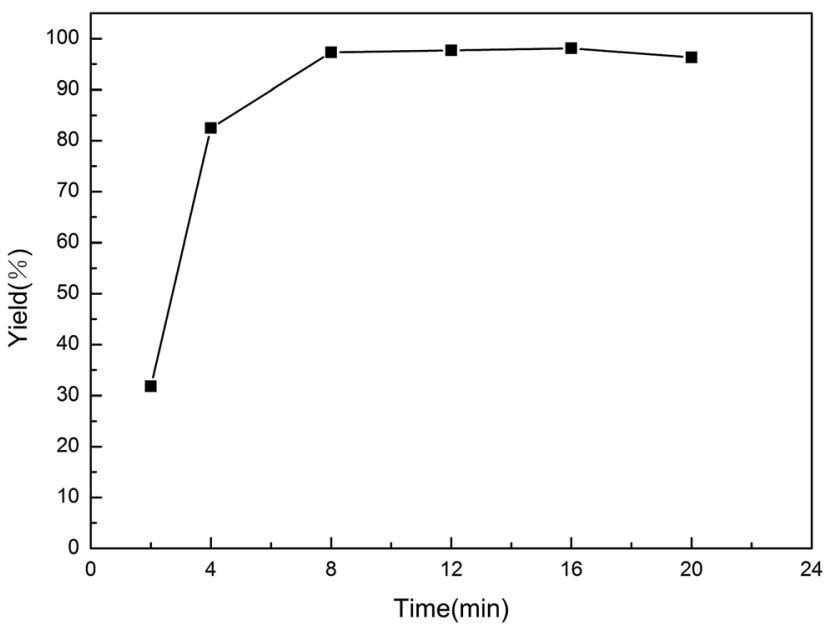

Fig. 5 Effect of reaction time on yield of $4 a$. Reactions were carried out on a $0.1 \mathrm{mmol}$ scale; reverse $\mathrm{ZnO}$ nanomicelles $20 \mathrm{~mol} \%$.

results showed the particle diameters of $\mathrm{ZnO}$ nanomicelles ranged from 120 to $250 \mathrm{~nm}$ and displayed a homogeneous size distribution after the five successive runs. The increasing $\zeta$ potential indicated the catalytic system tend to be stable gradually. No remarkable changes could be evidenced in the recovery of the catalytic system.

\section{Comparison of the substrates}

To explore the generality and scope of the protocol, the reaction of various aromatic aldehydes, amines and $\beta$-naphthol were extended to prepare a series of Betti base derivatives and the results are depicted in Table 5. It was found that both electronrich and electron-deficient aromatic aldehydes could be converted to the desired Betti base derivatives in the presence of reverse $\mathrm{ZnO}$ nanomicelles (10 mol\%) at $70{ }^{\circ} \mathrm{C}$ in $1 \mathrm{~h}$ in good yields (Table 5, 4a-4g). However, halogen or electronwithdrawing groups at meta position accelerated the reaction rather than electron-donating groups. Furthermore, the amine component of the three-component reaction was surveyed. To our delight, the reactions proceeded smoothly when aniline were used as substrate. Under the same reaction conditions, when piperidine was used as an amine partner, the desired product $4 \mathrm{~h}$ was obtained in $93 \%$ yield in 4 minutes (Table $5,4 \mathrm{~h}$ ). The results indicated that aliphatic amine and hydrazine were more active than aniline. In addition, a variety of Betti base derivatives were obtained in moderate yields when amide or
Table 5 Synthesized Betti base derivatives catalyzed by reverse $\mathrm{ZnO}$ nanomicelles

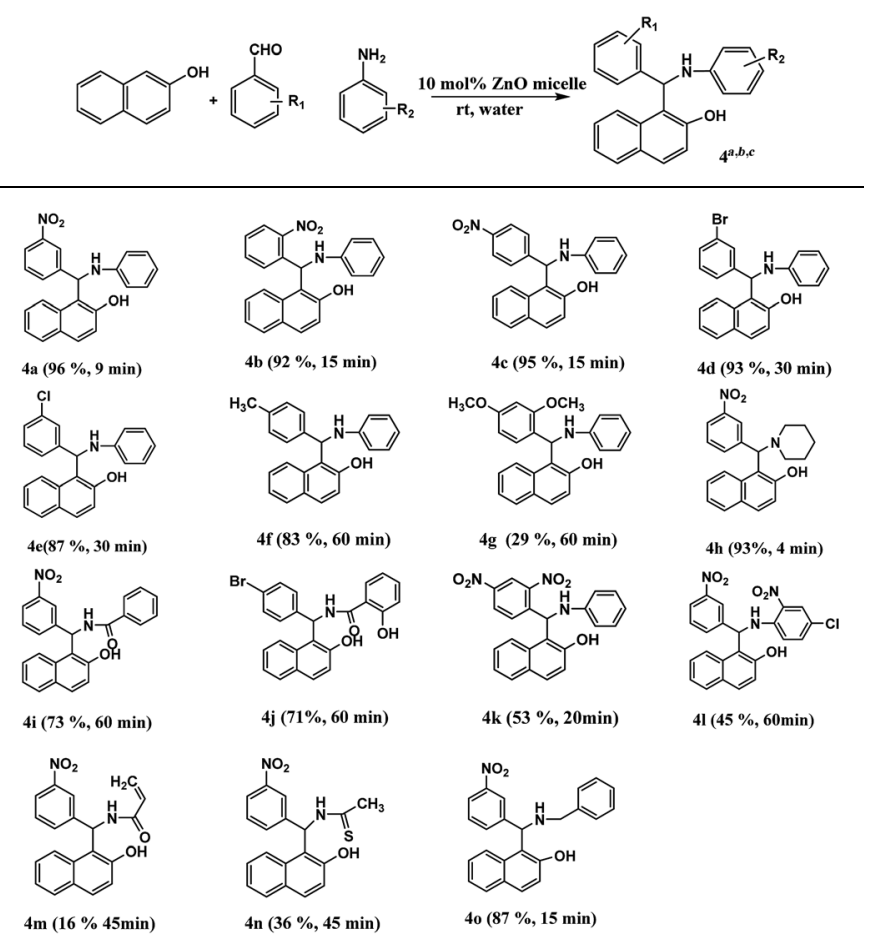

${ }^{a}$ Reactions were conducted with naphthol $(1.2 \mathrm{mmol})$, benzaldehyde ( $1 \mathrm{mmol})$, amine $(1 \mathrm{mmol})$ and reverse $\mathrm{ZnO}$ nanomicelles $(10 \mathrm{~mol} \%)$ in water $(10 \mathrm{~mL})$ at room temperature. ${ }^{b}$ The products were characterized by melting point, ${ }^{1} \mathrm{H},{ }^{13} \mathrm{C}$ NMR, IR and mass spectroscopy. ${ }^{c}$ Isolated yield.

thioacetamide were used as the substrates because the electron density of amino group was reduced by the electronwithdrawing conjugation effects of unsaturated bonds (Table $5,4 \mathrm{i}, 4 \mathrm{j}, 4 \mathrm{~m}$ and $4 \mathrm{n})$.

\section{Exploration of the possible mechanism}

The mechanism of this multi-component reaction involves a Knoevenagel condensation/Michael addition cascade process ${ }^{40} \mathrm{~A}$ tentative mechanism for the zinc oxide-catalyzed Mannich is proposed in Scheme 2 in the revised manuscript. Firstly, ZnO coordinates to benzaldehyde and generates the zinc oxide-aldehyde intermediate $\mathbf{1}$, which reacts with aniline to

Table 4 The reuse of reverse $\mathrm{ZnO}$ micelles in the synthesis of $4 \mathrm{a}^{a}$

\begin{tabular}{|c|c|c|c|c|c|c|c|}
\hline Cycle & Fresh & Reuse 1 & Reuse 2 & Reuse 3 & Reuse 4 & Reuse 5 & Reuse 6 \\
\hline Yield $^{b}(\%)$ & 96 & 95 & 96 & 97 & 95 & 95 & 94 \\
\hline Diameter (nm) & $116 \pm 12$ & $119 \pm 13$ & $142 \pm 13$ & $166 \pm 15$ & $186 \pm 14$ & $216 \pm 17$ & $251 \pm 15$ \\
\hline
\end{tabular}

${ }^{a}$ Conditions: 1 (1.2 mmol), 2a ( $\left.1 \mathrm{mmol}\right), 3 \mathrm{a}(1 \mathrm{mmol})$, catalyst (reverse ZnO nanomicelles, $\left.10 \mathrm{~mol} \%\right)$, solvent $($ water, $10 \mathrm{~mL}), 70{ }^{\circ} \mathrm{C}, 1 \mathrm{~h} .{ }^{b}$ Isolated yield. 


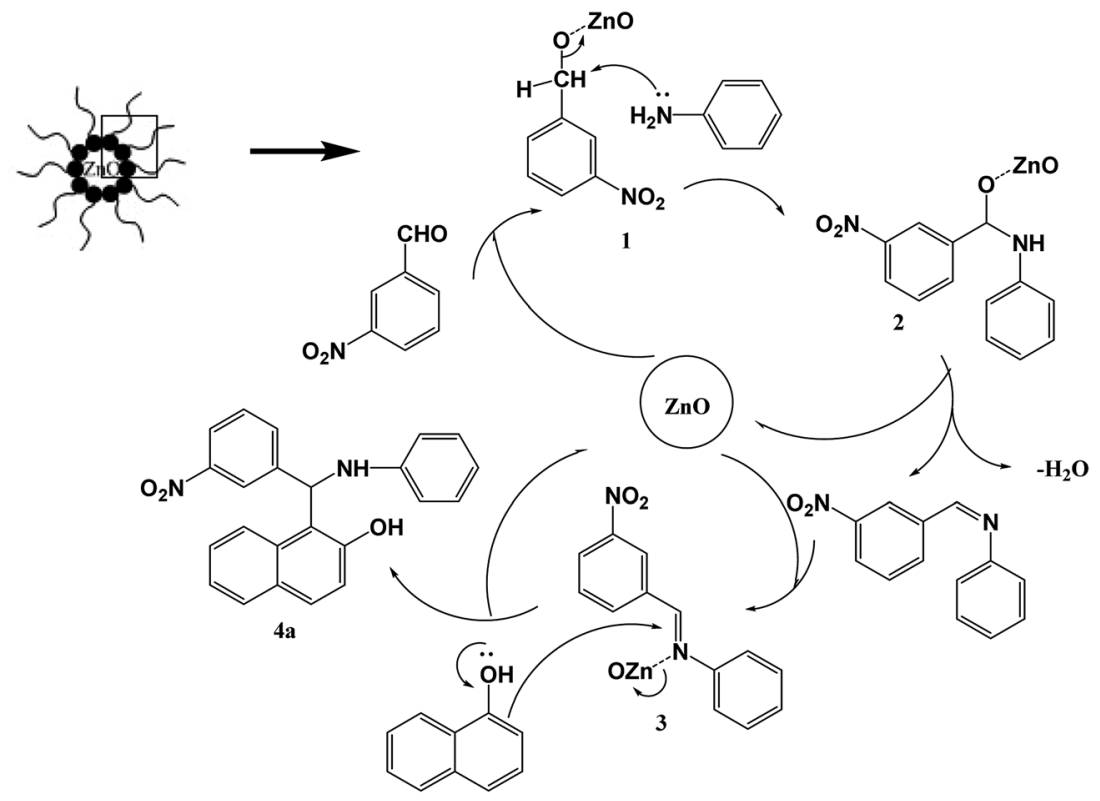

Scheme 2 Proposed mechanism for the synthesis of $4 a$.

generate the imine intermediate $\mathbf{2}$ after the dehydration and remove of zinc oxide. Subsequently, the imine intermediate is activated when $\mathrm{ZnO}$ binds to the nitrogen atom 3 which promotes the following nucleophilic addition of $\beta$-naphthol to give the desired product $\mathbf{4}$ and regenerates zinc oxide. Based on the results obtained, the formation of the imine intermediate is likely the rate-determining step. $\mathrm{ZnO}$, which is a Lewis acid, provides efficient acidic sites to form coordinate bond between oxygen atom (or nitrogen atom) which can activate the formation of imine intermediate, followed by facilitating the nucleophilic addition. Reverse micelles played an important role in combining the substrates, stabilizing the imine intermediate and promoting the dehydration condensation under neutral conditions in water catalyzed by the cationic water pool encapsulated in reverse micelles. The enhanced surface area due to nano particle size is an added advantage for its reactivity. Water promotes the nucleophilic addition reaction between imine intermediate and $\beta$-naphthol for the high polarity. All these important factors are responsible for the high accessibility of the substrate molecules on the catalyst surface. Thus, the reaction occurred more easily in a micelles special with respect to its functioning as a nanoreactor.

\section{Conclusions}

In conclusion, we have developed an efficient synthesis of Betti bases via the reaction of naphthols, aldehydes with amines in aqueous media. Reverse $\mathrm{ZnO}$ nanomicelles effectively catalyzed Betti reaction in excellent yields without the formation of any other by-product. This method has the advantages of atom economy and environmentally benign. The results prove the crucial role of reverse $\mathrm{ZnO}$ micelles as nanoreactors and will find more extensive applications in the field of green chemistry.

\section{Acknowledgements}

We are grateful for financial support by National natural science foundation of China (No. 81572349), the Natural Science Foundation of Jiangsu Province (No. BK20141149, BK20140225) the Science and technology project of Xuzhou city (No. KC16SY152) and the Director Found of the Jiangsu Province Key laboratory of New Drug Research and Clinic Pharmacy (ZR$\mathrm{XY} 201403)$. This project was also sponsored by Innovation Project of Xuzhou Medical College.

\section{References}

1 H. Gao, J. Sun and C. G. Yan, Chin. Chem. Lett., 2014, 26, 353. 2 C. Cardellicchio, M. A. M. Capozzi and F. Naso, Tetrahedron: Asymmetry, 2010, 21, 507.

3 A. Kumar, M. K. Gupta and M. Kumar, ChemInform, 2010, 41, 1582.

4 A. Y. Shen, C. T. Tsai and C. L. Chen, Eur. J. Med. Chem., 1999, 34, 877.

5 I. Szatmäri and F. Fülöp, Curr. Org. Synth., 2004, 1, 155.

6 H. R. Shaterian and H. Yarahmadi, Tetrahedron Lett., 2008, 49, 1297.

7 H. Gao, J. Sun and C. G. Yan, Chin. Chem. Lett., 2014, 26, 353. 8 A. Decottignies, C. Len and A. Fihri, Chemsuschem, 2010, 5, 502. 9 C. Mukhopadhyay, S. Rana and R. J. Butcher, ARKIVOC, 2010, 12, 291.

10 I. Szatmári, A. Hetényi, L. Lázár and F. Fülöp, J. Heterocycl. Chem., 2004, 41, 367.

11 H. Gao, J. Sun and C. G. Yan, Chin. Chem. Lett., 2015, 26, 353. 
12 A. Decottignies, C. Len and A. Fihri, Chemsuschem, 2010, 5, 502.

13 T. Maegawa, Y. Kitamura, S. Sako, T. Udzu, S. Ai and A. Tanaka, Chemistry, 2007, 13, 5937.

14 S. Kobayashi, H. Ishitani, S. Komiyama, D. C. Oniciu and A. R. Katritzky, Tetrahedron Lett., 1996, 37, 3731.

15 H. Moghanian, A. Mobinikhaledi, A. G. Blackman and E. Sarough-Farahani, RSC Adv., 2014, 4, 28176.

16 A. Kumar, M. K. Gupta and M. Kumar, Tetrahedron Lett., 2010, 51, 1582.

17 J. Auge, N. Lubin-Germain and J. Uziel, ChemInform, 2007, 38, 1739.

18 B. Karmakar and J. Banerji, Tetrahedron Lett., 2011, 52, 4957. 19 A. Shahrisa, R. Teimuri-Mofrad and M. GholamhosseiniNazari, Mol. Diversity, 2015, 19, 1.

20 A. Rezaeifard, P. Farshid, M. Jafarpour and G. K. Moghaddam, RSC Adv., 2014, 4, 9189.

21 A. Kołodziejczak-Radzimska, E. Markiewicz and T. Jesionowski, J. Nanomater., 2012, 20, 5603.

22 B. Karmakar and J. Banerji, Tetrahedron Lett., 2011, 52, 4957.

23 A. Punnoose, K. Dodge, J. W. Rasmussen, J. Chess, D. Wingett and C. Anders, ACS Sustainable Chem. Eng., 2014, 2, 1666.

24 W. Jiang, B. Xu, J. Li, Q. Lin, X. Zeng and H. Chen, Int. J. Chem. Kinet., 2007, 39, 672-680.

25 F. Li, F. Feng, L. Yu and J. Xie, J. Solution Chem., 2014, 43, 1331-1343.

26 T. Dwars, E. Paetzold and G. Oehme, Angew. Chem., Int. Ed., 2005, 44, 7174 .
27 J. Zhang, L. D. Sun, X. C. Jiang, C. S. Liao and C. H. Yan, Cryst. Growth Des., 2003, 4, 309.

28 A. Manino, J. P. Wilcoxon, A. P. Sylwester and J. S. Kawola, American Chemical Society national meeting, Denver, CO, United States, 1993.

29 J. G. Liu, J. M. Xing, C. Shen, C. L. Yang and H. Z. Liu, Biochem. Eng. J., 2004, 21, 273.

30 R. Shen, J. G. Liu, J. M. Xing and H. Z. Liu, Chin. J. Process Eng., 2005, 5, 255.

31 H. Abdizadeh, Y. Vahidshad, H. R. Baharvandi and M. A. Baseri, International Conference on Ultrafine Grained and Nanostructured Materials, 2012, 5, 559.

32 C. J. Li and L. Chen, Chem. Soc. Rev., 2006, 35, 68.

33 T. Murase, Y. Nishijima and M. Fujita, J. Am. Chem. Soc., 2012, 134, 162.

34 Y. Mori, K. Kakumoto and K. Manabe, ChemInform, 2000, 41, 3107.

35 K. Manabe, X. M. Sun and S. Kobayashi, J. Am. Chem. Soc., 2001, 123, 10101.

36 K. Suzuki, Pure Appl. Chem., 1994, 66, 1557.

37 P. Cotanda, A. Lu, J. P. Patterson, N. Petzetakis and R. K. O'Reilly, Macromolecules, 2012, 45, 2377.

38 H. Firouzabadi, N. Iranpoor and A. Jafari, Adv. Synth. Catal., 2005, 347, 655.

39 I. R. Piletic, D. E. Moilanen, D. B. Spry, N. E. Levinger and M. D. Faye, J. Phys. Chem. A, 2006, 110, 4985.

40 P. Heidari, R. Cheraghali and H. Veisi, Appl. Organomet. Chem., 2016, 30, 991. 